\title{
Intelligent Control System for Plugging Indicator
}

\author{
Patankar.M.K, Murali.N, Ilango Sambasivan.S ,Swaminathan.P \\ Electronics and Instrumentation Division \\ Indira Gandhi Centre for Atomic Research, Kalpakkam, Tamil Nadu, India \\ E-mail : patankar@igcar.gov.in
}

\section{Abstract}

An intelligent control system for plugging indicator has been developed and successfully implemented to measure the impurities level in liquid sodium. A firing angle based silicon controlled rectifier (SCR) power controller (phase angle) based method has been adopted to vary the speed of the plugging indicator (PI) blower to do the automation along with application software written in Turbo C 3.0. On execution of this software, control system finds the plugging temperature corectorsponding to $80 \%$ of normal flow of the sodium and deduced the impurities in sodium using the mathematical relations. This integrated control system supports both continuous as well as discontinuous mode of operation.

Key words : Breeder reactor, DACs, phase angle

\section{INTRODUTION}

Plugging Indicator is a device for monitoring the impurities i.e. oxides \& hydrides level in liquid sodium. It is used to measure the temperature at which an impurity precipitates or re-dissolves, i.e. the saturation temperature. The impurity concentration may be deduced from a known solubility/ temperature relationship. Plugging indicators are used in experimental sodium systems and in fast breeder reactor (FBR) sodium systems.

\section{TECHNICAL DESCRIPTION}

\section{Why sodium?}

Liquid sodium is used as coolant to transfer the heat produced in a fast breeder reactor core to steam water circuit. Metallic sodium is preferred for fast breeder reactor due to its following properties:-

1. Low neutron capture cross section.

2. High thermal conductivity viz $52 \mathrm{kcal} / \mathrm{hr}-\mathrm{m}-{ }^{\circ} \mathrm{C}$ which is approx 80 times that of water.

3. Low vapor pressure permitting use of thin wall vessels.

4. High boiling points (about $880{ }^{\circ} \mathrm{C}$ ) permitting high coolant outlet temperature.

5. Lower cost compared to other liquid metals.

Because of its high chemical activity liquid sodium tends to get contaminated with impurities such as oxygen and hydrogen contributed by "dirt" left over in piping and vessels. Thus grease, weld slag, surface oxides and moisture can be major sources of impurities within the system. Sources of impurities during the system operation are water, impure cover ages and atmosphere. In reactor grade sodium, oxide and hydrides impurities level should be less than $3 \mathrm{ppm}$ and $1 \mathrm{ppm}$ respectively and is considered as pure sodium.

\section{Effects of impurities}

The presence of impurities can cause several harmful effects to nuclear heat transfer systems. Oxides and hydrides are major impurities among others in liquid sodium. The presence of oxygen in liquid sodium above certain concentration can accelerate corrosion of stainless steel used in sodium systems. Hydrogen presence in primary sodium circuits tends to increase the reactivity whereas in secondary sodium circuit it indicates the sodium water reaction has occurred.

Thus, the above mentioned impurity concentrations are measured by the plugging indicator to ascertain whether sodium is pure or not.

\section{Plugging indicator setup}

The plugging indicator consists of an economizer, finned tube for cooling the sodium, permanent magnet (PM) flow meter for measuring sodium flow, orifice, thermo well with K-type thermocouple to measure the sodium temperature, $\mathrm{PI}$ blower to cool the sodium at orifice. Following Fig. 1 shows the details of plugging indicator.

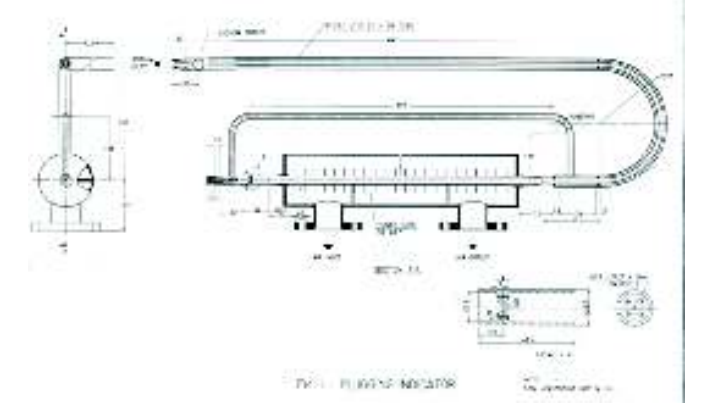

Fig. 1. Plugging indicator 


\section{Principle}

Plugging indicator is based on the temperature dependent solubility. Solubility of impurities decrease sharply with the fall in the temperature of sodium. As the temperature of sodium is decreased the impurities in the sodium gets solidifies. This property is used to know the impurities level in liquid sodium.

\section{Function}

Plugging indicator normally operates at rated flow and PI blower remains in off condition. For starting plugging run operation, PI blower has to be switched on and the sodium temperature at orifice outlet has to be cooled from normal temperature to $200^{\circ} \mathrm{C}$ at the cooling rate $5^{\circ} \mathrm{C} / \mathrm{min}$. After $200^{\circ} \mathrm{C}$, the cooling rate has to be reduced to around $2^{\circ} \mathrm{C} / \mathrm{min}$. When the temperature reaches saturation level of impurities that have temperature dependent solubility, these impurities will precipitate and gets deposited on the narrow orifice. The temperature corresponds to the initiation of deposit or flow reduction will be the saturation temperature. PI blower continuous cools the sodium as per specified cooling rate and flow at the orifice gets reduced. When flow reaches $80 \%$ of the normal flow, PI blower will be switched off and further sodium cooling will be stopped to avoid further flow reduction. The corresponding temperature is noted which is called as plugging temperature. After stopping the PI blower, the temperature will gradually increase and at a particular temperature of orifice, flow will attain the original value and temperature. This temperature is known as plug release temperature and is noted down. Thereafter, said impurities level can be determined with the help of following mathematical equations using plugging temperature and plug release temperature.

Log [Oxygen W ppm] $=6.257-(2444.5 / T)$. Log [Hydrogen W ppm] $=6.467-(3023 / T)$.

$$
\mathrm{T}=\text { Temperature in Kelvin }
$$

\section{Need of automation}

Plugging indicator operates manually from control room. This is nothing but an open loop plugging indicator. In this mode of operation, operator monitors the operation range for sodium flow and temperature, then switch on the PIblower. To cool the sodium, operator varies the voltage via variable transformer, which in turn varies the speed of the blower based on the current temperature reading. PI blower sucks and injects surrounding air into PI chamber. In this way operator cools the sodium as per asked cooling rate. When sodium flow gets reduced to $80 \%$ of the normal flow, operator switches off the PI blower and noted down the current temperature i.e. plugging temperature. After switching off the PI blower, flow restores the original flow and temperature and noted down the plug release temperature. Finally, based on these temperatures the operator manually calculates the impurity level in sodium with the help of equation (1) and (2).

Since the manual mode of operation has the several difficulties such as to maintain the asking cooling rate, macro step size for voltage of variable transformer so that smooth control over PI blower, accurate plugging point, continuous monitoring of temperature and flow signals, calculating the impurities manually, focused attention on plugging run operation, doesn't support the continuous mode of operation. To avoid the above major shortcomings, an intelligent control system is needed for plugging indicator. This intelligent control system can be operated automatically to facilitate the operator to overcome the above-mentioned difficulties in running plugging indicator. The developed automatic plugging indicator has both continuous mode and discontinuous mode of operation.

\section{Continuous mode}

In continuous mode, the PI blower will be operate continuously and sodium flow will be maintained at $80 \%$ (selectable from $7095 \%$ ) of the original flow by continuous cooling of the orifice. The orifice temperature measured is the saturation temperature and this value is available continuously. This mode of operation maintains the stable partial plug of impurity in the orifice. Ingress of hydrogen orother impurity will give rise to increase in saturation temperature and PI blower will be controlled to maintain the sodium flow at specified rate to measure the saturation temperature continuously.

\section{Discontinuous mode}

In discontinuous mode, whenever operator wants to know the impurity level in sodium, the PI blower will be switched on and the orifice will be cooled at prescribed cooing rate. The plugging temperature and plug release temperature will be measured and corresponding impurity level will be determined with the help of equation (1) and (2). The time interval shall be selectable.

\section{AUTOMATION METHODOLOGY}

For automation of plugging indicator, an intelligent control system has been developed. Controlling the speed of the PI blower to maintain the accurate cooling rate is the core idea behind of the 
automaton. SCR power controllers provide a relatively economical means of power control. SCR power controllers cost less and more efficient than variable transformers. Compared to contactors, SCR power controllers offer a much finer degree of control and do not suffer from the maintenance problems of mechanical devices. Feature and benefits of SCR power controllers over other forms of control include:infinite resolution, extremely fast response, no moving parts, less noise, less space, higher reliability and long life. SCR power controllers have following two basic control modes: -

\section{Phase angle mode}

In phase angle mode, the power is transmitted to the load is controlled by firing the SCR for a part of the power supply voltage half cycle. SCR can deliver the variable power from 0 to $100 \%$ to the load based upon the firing angle. The power is transmitted to the load is proportional to the input voltage but is not the linear function of the firing angle.. Since, using this mode smooth control over $\mathrm{PI}$ blower is possible, hence this mode has been adopted for automation of plugging indicator.

\section{Zero voltage switching mode}

In this control mode, SCRs are turned on only when the instantaneous value of the sinusoidal waveform is zero. In zero-cross operation, power is applied for a number of continuous half cycles and then removes for a number of cycles to achieve the desired load power. Thus, it transfers either full power or zero power to the load. This method is not suitable for controlling the speed of PI blower. Therefore, this approach has been dropped.

It is clear from above discussion that SCR power controller (phase angle) based method is ideal for automation of plugging indicator.

\section{Hardware architecture}

Fig. 2 shows the hardware architecture for SCR power controller (phase angle) based method for automation of plugging indicator. This is a close loop control system consisting of major elements such as ADAM-5510, SCR power controller and PI blower.

\section{ADAM-5510}

Advantech make ADAM-5510 is ideal for PC based data acquisition and control system (DACS) applications. It isa standalone compact PC with an Intel x86- CPU running onDatalight based ROM-DOS. It provides flash ROM about 171KB for application software. It communicates with PC via RS-232, 3-wire interface. It also provides well-stocked C libraries, including system resources, functions, I/O functions, popular control algorithms and communication functions. Application software can be written in Turbo $\mathrm{C}$ 3.0 and their executable file is downloaded in the flash memory. Since user application software sits in the ADAM-5510's flash ROM, therefore the virus cannot affect it or tempering is also not possible. ADAM- 5510 can handle up to 4 I/O slots. ADAM- 55010 along with I/O modules operates on $24 \mathrm{Vdc}$ unregulated power supply, which is provided by the PWR-243 unit (not shown in the Fig. 2). PWR-243 accepts the 230Vac, 1- $\phi$ power supply from plant and generates the unregulated power supply for operation of ADAM- 5510 .

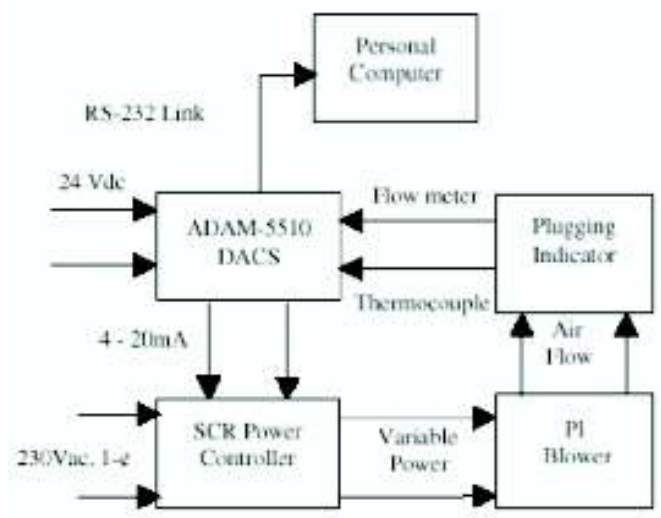

\section{Fig. 2.SCRPower controller (PhaseAngle)based method}

Analog input module ADAM5018 (2 Nos) and analog output module ADAM5024 (1 No.) has been selected based on $1 / 0$ requirement. These all modules housed in ADAM05510's I/O slots. ADAM5018 is a7-channel differential thermocouple module which can accepts $\mathrm{mV}, \mathrm{mA}, \mathrm{V}$ and thermocouple inputs. Out of two ADAM-5018 modules, one module is configured for K-type thermocouple to handle sodium temperature signal whereas other one is configured for $\mathrm{mV}$ signal to handle flow signal. Unused channels of ADAM-5018 modules are disabled. In ADAM- 5018 (forthermocouple) one channel is reserved for taking care of cold junction compensation. ADAM-5018 modules can samples the signals at 10 sample/sec with accuracy $0.1 \%$. It provides the fault and over voltage protection up to $\pm 35 \mathrm{~V}$. ADAM5024 analog output module configured for 4- $20 \mathrm{~mA}$ signal. This module can generates the analog current output with $\pm 0.1 \%$ of fullscale range (FSR) and resolution is $0.0015 \%$ of FSR.

\section{SCR Power controller}

Eurotherm make SCR power controller (phase angle)425A has been selected to control the speed of PI 
blower. It operates on 230Vac, single-phase power supply. SCR power controller can accept control signal in form of analog dc current $(4-20 \mathrm{~mA})$ as well as in analog dc voltage $(0-10 \mathrm{~V})$. The capacity of this unit is 2500 watts. No cooling fan is needed and power /current delivers to the PI blower can be limit. This controller is interfaced with the ADAM-5510. The PI blower. The PI blower sucks the surrounding air and injects into the plugginh indicator's cooling chamber to cool the sodium.PI blower operates on 230Vac,1- $\phi$ power supply and the capacity of PI blower is 750 watts. Airflow rate of PI blower is $200 \mathrm{~m}^{3} / \mathrm{hr}$.

In Fig. 2. control system elements are arranged in planned to perform the plugging run operation. ADAM5510 is the heart of the control system in which thermocouple signal $\left(0-600^{\circ} \mathrm{C}\right)$ is wired to the ADAM 5018 (thermocouple module) whereas flow signal $(0-4 \mathrm{mV})$ is routed to other ADAM-5018 (configured for $\mathrm{mV}$ signal). Analog output signal from ADAM-5024 is connected to the SCR power controller which controls the power delivered to the PI blower as per available control signal.

\section{Software architecture}

To alive the control system, application software has been written in Turbo $C$ 3.0. For writing the application software $1 / 0$ libraries to handle thermocouple, flow and analog DC output signals, commutation functions for communicating with PC provided by the Advantech are included along with C libraries. After developing the application software, their executable file has been generated and downloaded in the ADAM-5510's flash memory and executed from PC (in terminal mode). This software supports both continuous as well as discontinuous mode of operation. Flowchart for discontinuous and continuous mode of operation has been at abstract level is shown in Fig. 3 and 4 respectively.

\section{OPERATION}

Integrated control system has been installed, tested and commissioned in experimental sodium system of engineering Hall-III of IGCAR. Both continuous and discontinuous mode of operation is tested one by one.

\section{Discontinuous mode of operation}

Whenever operator wants to know the impurities level in liquid sodium, operator executes the application software of discontinuous mode of operation. To initiate the plugging run operator presses the $Y$ key from the keyboard, then immediately application software starts plugging run. Firstly, application software scans the sodium temperature and flow signals via ADAM-5018 modules and initiates the action to check whether both signals values are stable or not i.e. consistency and operating range for above signals. If everything is ok as per requirement, then application software generates control signal via ADAMO5024 module in form of current $4-20 \mathrm{~mA}$, which in turn delivers the power to the PI blower via SCR power controller. Thus PI blower starts to cools the sodium at $5^{\circ} \mathrm{C} / \mathrm{min}$ if sodium temperature is above than $200^{\circ} \mathrm{C}$ otherwise cools the sodium at $2^{\circ} \mathrm{C} / \mathrm{min}$. Thus application software continuous monitors the temperature and flow signals continuously and based on that, it does the processing as per logic, generates the control signal to vary the speed of the PI blower to cool the sodium as per said cooling rate.

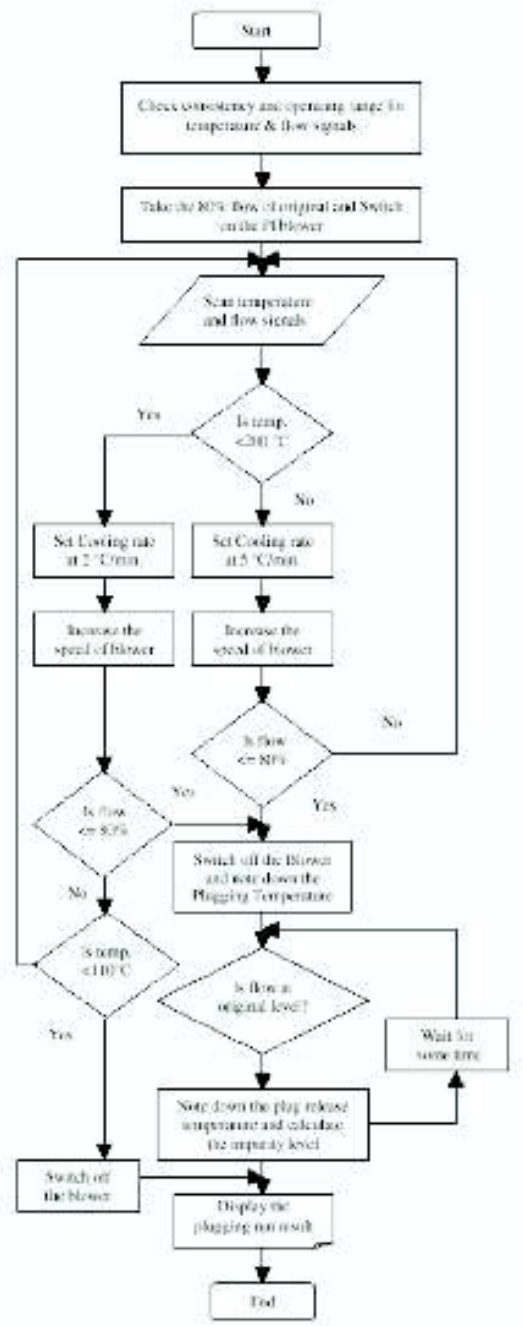

Fig. 3. Flowchart for discontinuous mode of plugging indicator 


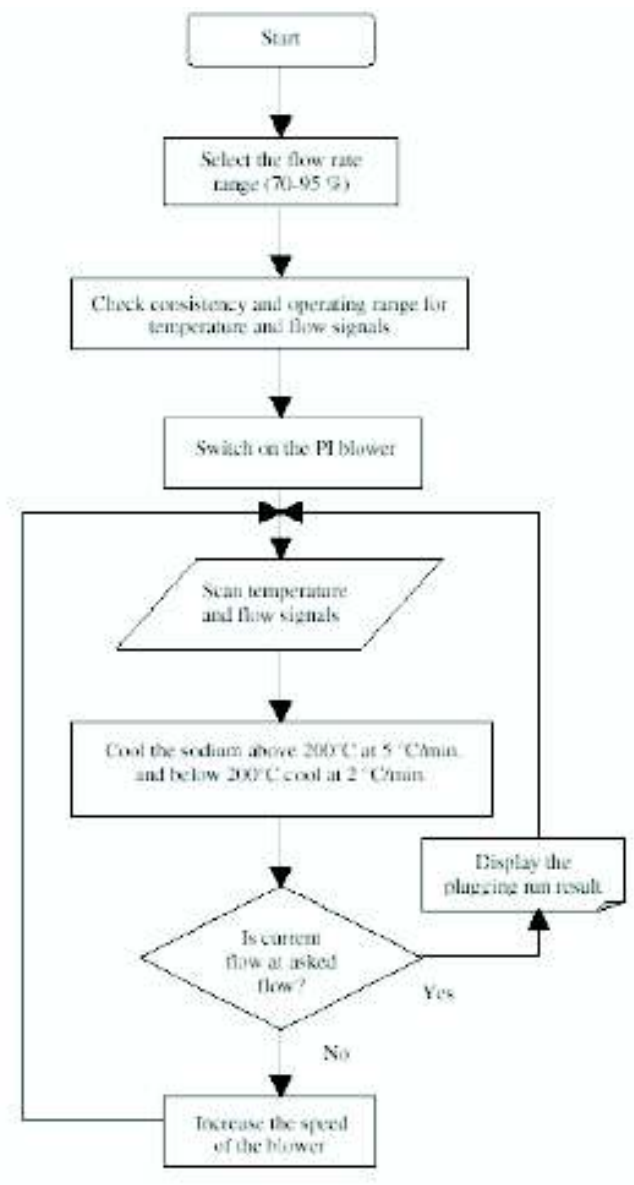

Fig. 4. Flowchart for continuous mode of plugging indcator

At some point of the time, when sodium flow reduced to $80 \%$ of the normal flow, then application software gives the control signal to switch off the PI blower and acquires the corresponding temperature, which is nothing but the plugging temperature. Still, application software continuously monitors the temperature and flow. When flow attains the original flow, then application software also note the corresponding current temperature, which is the plug release temperature. Based on plugging and plug release temperature, software does the mathematical calculations using equation (1) and (2) and find out the oxygen and hydrogen impurities level in sodium. At the end, plugging run results are displays in particular format on PCs.

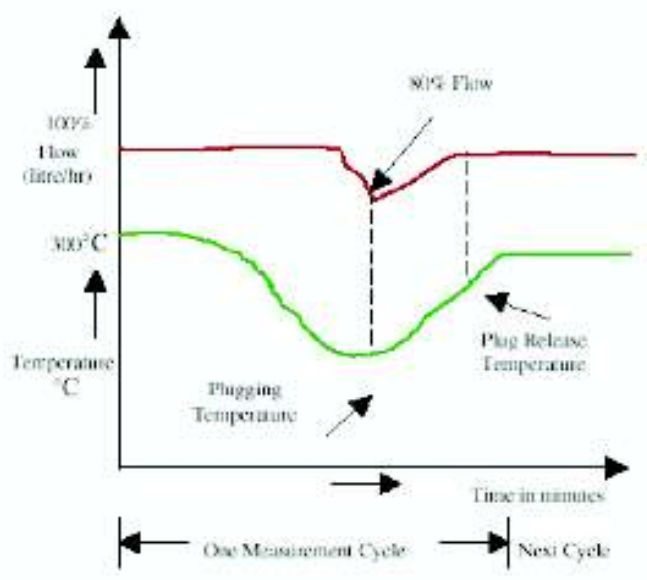

Fig. 5. Discontinuous mode of plugging indicator if sodium is impure

Fig. 5. shows the curve for temperature and flow value with respect to time for one measurement cycle. This curve shows the impurity present in the sodium. These curves show the plugging and plug release temperature.If sodium is pure then there is not flow reduction in the orifice, then it is assumed that the sodium is pure. Atbelow $110^{\circ} \mathrm{C}$ sodium, this control system immediately switches off the PI blower. Below $110^{\circ} \mathrm{C}$ sodium cooling is not allowed because the melting point of sodium is $98^{\circ} \mathrm{C}$. Fig. 6 shows sodium temperature and flow behavior in case there is no impurity in sodium. In this situation, sodium flow curve is a straight line i.e. no fluctuation in sodium flow

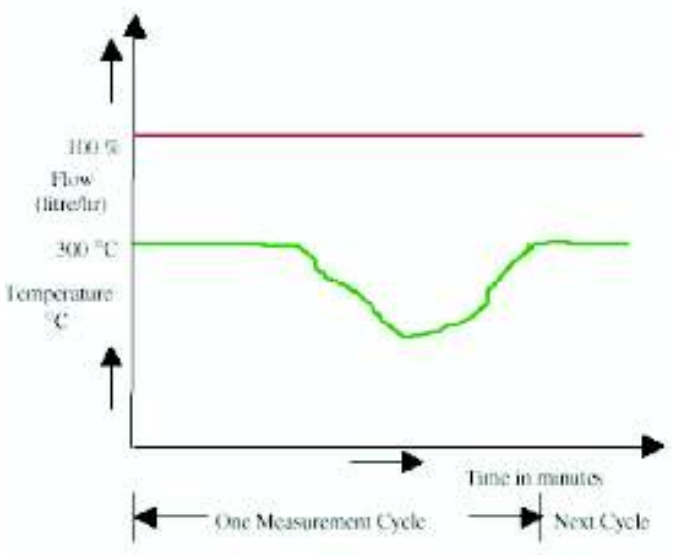

Fig. 6. Discontinuous mode plugging indicator if sodium is pure 


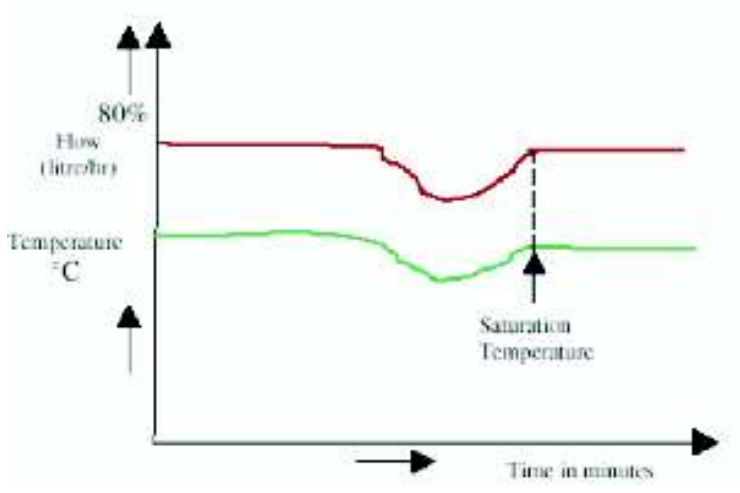

Fig. 7. Continuous mode of plugging indicator

\section{Continuous mode of operation}

When this mode of operation executes from the terminal mode of PC, application software ask to select the sodium flow range from $70-95 \%$. After selecting the flow rate, application software acquires the temperature \& flow value and checks the consistency \& operation range for same signals. If everything is ok then software gives the command to switch on the PI blower. Application software scans the temperature \& flow signals, process the logic as per requirement and gives the control signal 4-20mA current for SCR power controller, which in turn varies the speed of the PI blower.

Thus PI blower starts to cool the sodium at asked cooling rate depend upon the current temperature When sodium flow becomes equal to the selected flow then control system continuously monitors the both signals, varies the blower speed and maintains the partial plug condition at the orifice at selected flow.If impurities ingress in the sodium, flow and temperature sharply gets reduced then $\mathrm{Pl}$ blower reduced the blower speed so that flow once again come to the original flow and note down the corresponding temperature. Corresponding to this temperature, which is the saturation temperature, software calculates the impurity level in sodium and displays the results on the asking format. Thus this control system continuously looks for the saturation temperature. Fig. 7 shows the curve between sodium temperature and flow value and time for continuous mode of operation.

\section{ACKNOWLEDGEMENT}

The authors acknowledge Mr. K. K. Rajan, Head Sodium Technology Division for supporting and guiding to execute this project. The authors also thank his colleagues for their suggestions in the preparation of this article.

\section{REFERENCES}

[1] K.K. Rajan, "Requirement Specification Document for Plugging Indicator Intelligent Control System" EDG/STD/99130/DN/3005.

[2] Preliminary Safety Analysis Report Chapter 6 Heat Transport Sodium Circuits 